\title{
CALCULATION OF CHARGE-CHANGING CROSS SECTIONS OF IONS OR ATOMS COLLIDING WITH FAST IONS USING THE CLASSICAL TRAJECTORY METHOD*
}

\author{
I. D. Kaganovich, A. Shnidman, H. Mebane, and R.C. Davidson \\ Plasma Physics Laboratory, Princeton University, Princeton, New Jersey 08543, U.S.A
}

\begin{abstract}
Evaluation of ion-atom charge-changing cross sections is needed for many accelerator applications. A classical trajectory Monte Carlo (CTMC) simulation has been used to calculate ionization and charge exchange cross sections. The results of simulations using the CTMC method for the ionization and charge exchange cross sections for the interaction of $1 \mathrm{MeV} \mathrm{K}^{+}$with $\mathrm{H}_{2}, \mathrm{He}$, and $\mathrm{Ne}$ are presented. The simulated values of the total cross section agree well with the experimental data.
\end{abstract}

\section{INTRODUCTION}

Ion-atom ionizing collisions are of considerable interest in atomic physics [1] and play an important role in many applications, such as heavy ion inertial fusion [2], collisional and radioactive processes in the Earth's upper atmosphere [3], atomic spectroscopy, ion stopping in matter, and ion-beam lifetimes in accelerators [4]. For example, electron clouds can form inside the accelerator due to residual gas ionization and cause two-stream instabilities [5]. Formation of the electron clouds and the beam loss due to stripping can cause severe limitations on parameters of the vacuum system for the heavy ion synchrotron SIS18 at GSI operating with heavy ion beams [6]. Beam interaction with the remaining background gas and gas desorbing from the walls can limit the charge bunch intensity at the Relativistic Heavy Ion collider (RHIC) [7], and is also a concern for the Large Hadron Collider (LHC) [8]. Similarly, it is of considerable concern for the positron damping ring of the International Linear Collider (ILC) [9], as well as for other high-current, high-intensity accelerators and ion beam injectors.

To estimate the ionization and stripping rates of fast ions propagating through gas or plasma, the values of ion-atom ionization cross sections are necessary. In contrast to the electron and proton ionization cross sections, where experimental data or theoretical calculations exist for practically any ion or atom, the knowledge of ionization cross sections by fast complex ions and atoms is far from complete. For this reason the U.S. Heavy Ion Fusion Science Virtual National Laboratory has initiated measurements of cross sections in a series of experiments at GSI $[10,11,12]$ and the Texas A\&M synchrotron [13]. When experimental data and theoretical calculations are not available,

\footnotetext{
*Work supported by the U.S. Department of Energy and the National Undergraduate Fellowship program.

\#ikaganov@pppl.gov
}

approximate formulae are needed; therefore, the scaling of cross sections with energy and target or projectile nucleus charge have been developed to approximate the values of the cross sections over a broad range of energies and charge states $[1,11,14]$.

For the interaction of complex projectile and target atoms or ions, classical trajectory Monte Carlo (CTMC) simulations can be utilized. Classical mechanics approaches are typically simple to apply and yield fairly reliable total cross sections for collision processes at intermediate energies [15].

\section{DESCRIPTION OF THE CTMC METHOD}

Application of the CTMC method consists of computing of the electron trajectory in an atom when another ion or atom is passing by at a certain impact parameter. For calculating the total cross section it is only necessary to determine the outcome of the collision, i.e., the electron velocity and distances to the target and projectile nuclei at large enough times, when one of the distances is sufficiently large. There are three possible outcomes: the electron remains close to one of the nuclei, or it moves far away from both of them. If the electron kinetic energy (in the appropriate reference frame) is smaller than the attractive potential of the target or projectile, the electron is assumed to be trapped by the nucleus. If the electron remains near the target, no ionization or charge exchange events have occurred. If the electron is trapped by the projectile nucleus, the exchange event has occurred. If none of these events has happened, ionization takes place. The results have to be averaged over all possible initial electron positions and impact parameters. Details of the method and results of the calculations for the ionization and charge exchange cross sections for collisions of various ion projectiles with hydrogen and helium targets are described in Refs. [16, 17].

\section{COMPARISON OF CTMC CALCULATIONS AND EXPERIEMNTAL DATA}

The cross sections for charge-changing collisions of fast potassium ions with different target atoms are needed to estimate the generation of electrons in the accelerator section of ion beams in the High Current eXperiment 
(HCX) and the Neutralized Drift Compression eXperiment (NDCX) at Lawrence Berkeley National Laboratory (LBNL) [2]. Therefore, these total cross sections have been measured in Ref. [18]. The sum of ionization and charge-exchange cross sections for several gas targets $\left(\mathrm{H}_{2}, \mathrm{~N}_{2}, \mathrm{He}, \mathrm{Ne}, \mathrm{Kr}, \mathrm{Xe}, \mathrm{Ar}\right.$, and water vapor) impacted by a $1 \mathrm{MeV} \mathrm{K}^{+}$beam were measured. In a high-current ion beam, the self-electric field of the beam is high enough that the ions produced from gas ionization or charge exchange by the ion beam are quickly swept aside in the accelerator. The flux of the expelled ions is measured by a retarding field analyzer. This allowed accurate measurements of the total charge-changing cross sections (ionization plus charge exchange) of the beam interaction with gas. The cross sections for $\mathrm{H}_{2}, \mathrm{He}$, and $\mathrm{N}_{2}$ have been simulated using the CTMC method and compared with the experimental results, showing very good agreement.

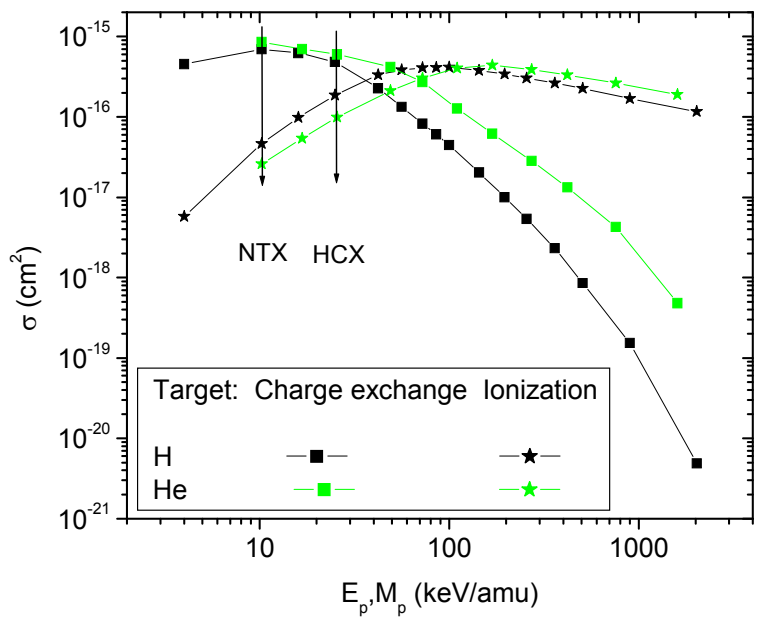

Figure 1. Charge exchange and ionization cross sections of atomic $\mathrm{H}$ and $\mathrm{He}$ target ions interacting with $\mathrm{K}^{+}$ions, predicted using CTMC calculations. The HCX parameters $\left(1 \mathrm{MeV} \mathrm{K}^{+}\right.$ion) correspond to $25 \mathrm{keV} / \mathrm{amu}$.

Figure 1 shows the CTMC theoretical prediction for charge-changing cross sections as a function of projectile energy. In the low-energy region, i.e., when the projectile velocity is much slower than the least tightly bound electron in the target molecule, the charge exchange process dominates over ionization. When the projectile velocity becomes much larger than the velocity of the least tightly bound electron in the target atom, the charge exchange cross section decreases rapidly [19]. The ionization cross section decreases with increasing projectile energy, approaching for large energies, the $(\ln E) / E$ dependence of the Bethe formula [1].

Therefore, in the high-energy region, i.e., when the projectile velocity is much larger than the least tightly bound electron in the target molecule, ionization dominates over the charge exchange mechanism and has a larger cross section.
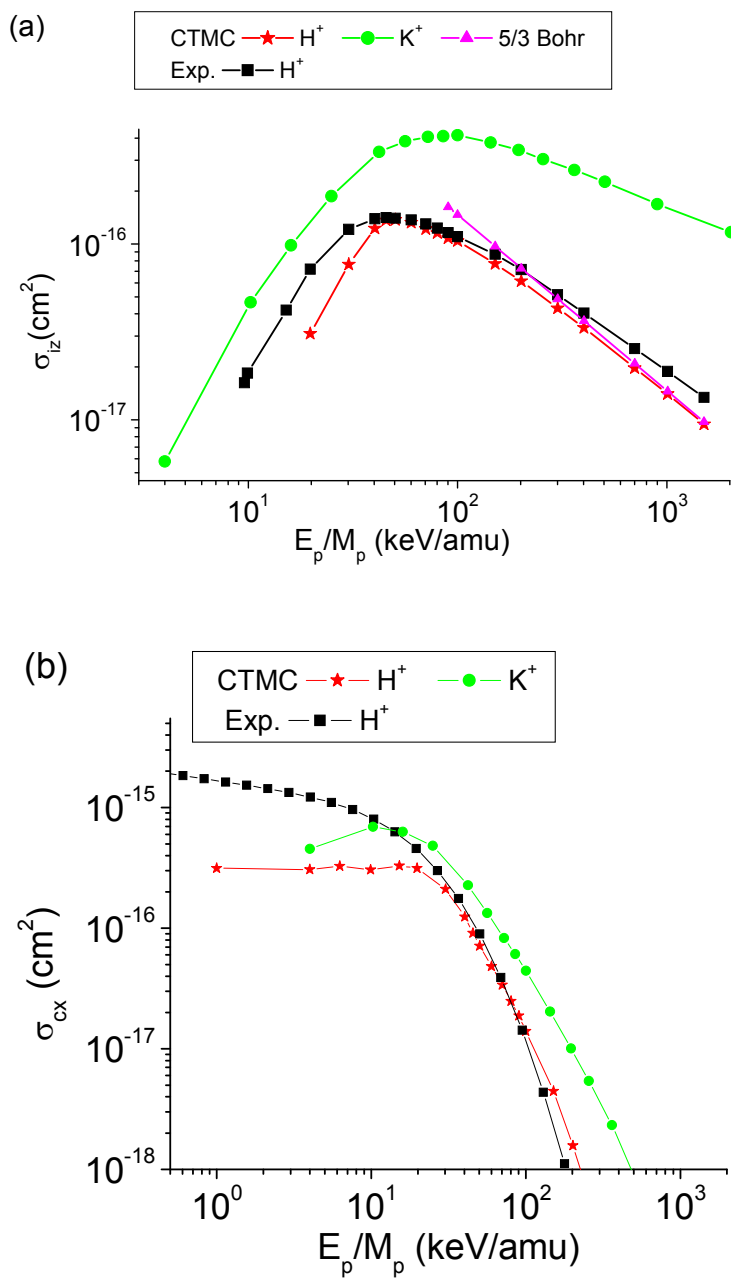

Figure 2. Comparison of ionization cross section (a) and (b) charge exchange cross sections for proton and potassium ion projectiles colliding with atomic hydrogen. The experimental data are from Refs. [1, 3].

Figure 2 illustrates that the contribution of collisions with impact parameter less than the potassium ion radius (inside the potassium ion) are important for cross section estimates, because the potassium ion cross sections are significantly larger than the proton cross sections. Therefore, it is important to accurately model the potassium ion atomic potential near the outer edge of the ion radius. The atomic potential of the potassium ion can be determined either by using Thomas-Fermi theory or Hartree-Fock theory, which include orbital effects. Calculations show that the Thomas-Fermi theory does not describe accurately the ion potential at the outer edge of the potassium ion. The difference in atomic potentials for singly-charged potassium ions is important, and gives an error of about $20 \%$ compared with the calculations utilizing the more accurate Slater model as shown in Table I.

The results of simulations using the CTMC method for the ionization and charge exchange cross sections for 
the interaction of $1 \mathrm{MeV} \mathrm{K}^{+}$with $\mathrm{H}_{2}, \mathrm{He}$, and $\mathrm{Ne}$ are summarized in Table I. For a $1 \mathrm{MeV} \mathrm{K}^{+}$beam, the values of the charge exchange cross sections are 2-4 times higher than the ionization cross sections; the total cross section agree well with the experimental data [18], as shown in Table II.

Table I: Ionization and charge exchange cross sections for the interaction of $1 \mathrm{MeV} \mathrm{K}^{+}$with $\mathrm{H}_{2}, \mathrm{He}$, and $\mathrm{Ne}$. TF denotes the calculation using the Thomas-Fermi model of the potassium ion, and Slater indicates the more accurate model based on the Hartree-Fock atomic wave equations solved by the use of Slater determinants [20].

\begin{tabular}{|c|c|c|c|c|}
\hline \multirow[t]{2}{*}{ Gas } & \multicolumn{2}{|c|}{$\begin{array}{l}\text { Charge Exchange } \\
\text { cross }\left(10^{-16} \mathrm{~cm}^{2}\right)\end{array}$} & \multicolumn{2}{|c|}{$\begin{array}{l}\text { Ionization cross } \\
\text { section }\left(10^{-16} \mathrm{~cm}^{2}\right)\end{array}$} \\
\hline & Slater & $\mathrm{TF}$ & Slater & $\mathrm{TF}$ \\
\hline $\mathrm{H}_{2}$ & 5.9 & 9.7 & 3.0 & 3.7 \\
\hline $\mathrm{He}$ & 4.1 & 6.0 & 1.1 & 0.99 \\
\hline
\end{tabular}

Table II: Comparison of the calculated values of the total cross sections (sum of the ionization and charge exchange cross sections) with the experimental data [18] for the interaction of $1 \mathrm{MeV} \mathrm{K}^{+}$with $\mathrm{H}_{2}, \mathrm{He}$, and $\mathrm{Ne}$.

\begin{tabular}{clc}
\hline Gas & $\begin{array}{c}\text { Experiment } \\
\left(10^{-16} \mathrm{~cm}^{2}\right)\end{array}$ & $\begin{array}{c}\text { CTMC, Slater } \\
\text { model }\left(10^{-16} \mathrm{~cm}^{2}\right)\end{array}$ \\
\hline $\mathrm{H}_{2}$ & $13.5 \pm 1.5$ & 8.9 \\
$\mathrm{He}$ & $5.62 \pm 0.57$ & 5.20 \\
$\mathrm{Ne}$ & $11.9 \pm 1.0$ & 13.4 \\
\hline
\end{tabular}

Straightforward application of multi electron atoms appeared to be impossible because the multi-electron atom is unstable classically [17].

\section{CONCLUSIONS}

As evident from the figures showing comparisons between the simulations and experimental data, the CTMC simulations match the experimental results for projectile velocities between and 1 and 3 atomic units, which correspond to the region near the maximum value of the cross section. The CTMC method can underestimate the value of the cross sections outside this velocity range. An effective algorithm needs to be developed to make sure that artificial auto-ionization in collisions of two electrons in classical mechanics does not contribute to charge-changing collisions.

\section{REFERENCES}

[1] I. D. Kaganovich, E. A. Startsev and R. C. Davidson, New Journal of Physics 8, 278 (2006).

[2] B. G. Logan, et al, Nucl. Instr. and Methods A 577, 1 (2007).

[3] H. -D. Betz, Rev. Mod. Phys. 44, 465 (1972).

[4] H. Beyer, V. P. Shevelko, Atomic Physics with Heavy Ions (Springer, Berlin, 1999).
[5] R. A. Bosch, Phys. Rev. ST Accel. Beams 6, 074201 (2003).

[6] A. Smolyakov, W. Fischer, C. Omet, and P. Spiller, GSI Report No. GSI-Accelerator-Report-2005-11001 (2005).

[7] W. Fischer, et al., Proc. Eur. Part. Acc. Conf., Paris, France, 2002, p. 1485.

[8] T. Demma, et al., Phys. Rev. ST Accel. Beams 10, 114401 (2007).

[9] L. Wang, F. Zimmermann, SLAC Report No. SLACPUB-12642, 2007.

[10] R. E. Olson et al, J. Phys. B 35, 1893 (2002); J. Phys. B: At. Mol. Phys. 37, 4539 (2004).

[11] R. L. Watson, et al, Phys. Rev. A 67, 022706 (2003).

[12] R. D. DuBois, et al, Physical Review A 70, 032712 (2004).

[13] D. Mueller, et al, Phys. Plasmas 8, 1753 (2001); Laser Part. Beams 20, 551 (2002).

[14] A. C. F. Santos and R. D. DuBois, Phys. Rev. A 69, 042709 (2004).

[15] G. Schiwietz and W. Fritsch, J. Phys. B: At. Mol. Phys. 20, 5463 (1987).

[16] A. Shnidman, I. D. Kaganovich, and R. C. Davidson, PAC 2007 Proceedings of the 2007 Particle Accelerator Conference, 3678-3680 (2007).

[17] I. D. Kaganovich, et. al., Nucl. Instr. and Methods A to be published (2009).

[18] M. Kireeff Covo, et al., Phys. Rev. A 78, 032709 (2008).

[19] R. K. Janev, L. P. Presnyakov, and V. P. Shevelko, Physics of Highly Charged Ions (Springer, Berlin, 1985).

[20] E. Clementi and C. Roetti, Atomic Data and Nuclear Data Tables, 14, 177 (1974). 